\title{
REDES INTERFEDERATIVAS DE SERVIÇOS DE SAÚDE E CONTROLE DO MINISTÉRIO PÚBLICO: O PAPEL DOS ESTADOS NO SUS
}

\author{
INTERFEDERATIVE NETWORKS OF HEALTH SERVICES AND PUBLIC \\ PROSECUTOR'S OFFICE CONTROL: THE ROLE OF THE STATES IN THE SUS
}

\begin{abstract}
Antonio Carlos Da Ponte
Procurador de Justiça do Ministério Público do Estado de São Paulo. Diretor do Centro de Estudos e Aperfeiçoamento Funcional - Escola Superior do Ministério Público (CEAFESMP). Mestre, Doutor e Livre Docente pela PUC-SP. Professor dos Programas de Graduação e Pós-Graduação da PUC-SP e da Universidade Santa Cecília (Unisanta).

E-mail: acdaponte@uol.com.br
\end{abstract}

Reynaldo Mapelli Júnior

Promotor de Justiça do Ministério Público do Estado de São Paulo. Assessor do Centro de Estudos e Aperfeiçoamento Funcional - Escola Superior do Ministério Público (CEAFESMP). Doutor em Ciências pela Faculdade de Medicina da Universidade de São Paulo (FMUSP). E-mail: reynaldomapelli@mpsp.mp.br

Recebido em: 30/08/2017

Aprovado em: 15/11/2017

Doi: $10.5585 /$ rdb.v18i7.790

\begin{abstract}
RESUMO: O presente artigo possui como objeto de pesquisa o Sistema Único de Saúde (SUS). Criado pela Constituição Federal de 1988, que lhe dedicou um capítulo específico, o SUS avançou consideravelmente em políticas destinadas a garantir o direito à saúde a todos que residem no Brasil. Apesar de avanços, muitos problemas ainda persistem e continua a luta pelo SUS, cujos princípios e diretrizes constitucionais dependem da implementação gradual e adequada de serviços que respeitem o regime jurídico constitucional do SUS, sem privilégios e distorções. O objetivo do artigo é verificar a atuação do Ministério Público na fiscalização do papel que o Estado-membro pode e deve exercer na organização do SUS, em busca do atendimento integral da população, de acordo com os parâmetros constitucionais e legais. Assim, apesar da municipalização das ações e serviços de saúde, é essencial que o Estado-membro desempenhe, em respeito à Constituição Federal e à Lei Orgânica da Saúde, as atribuições de indutor da organização regionalizada e promotor de ações sanitárias complementares, contribuindo para a consolidação do SUS, o que deve ser objeto de controle do Ministério Público.
\end{abstract}

Palavras-Chave: Sistema Único de Saúde; Ministério Público; direito à saúde.

ABSTRACT: This article has as research object the Unified Health System (SUS, in portuguese). Created by the Federal Constitution of 1988, with a special dedicated chapter, SUS improved considerably in policies to guarantee the right for health for all residents in Brazil. Despite advances, many problems still persist and the struggle for SUS continues, whose constitutional principles and guidelines depends on the gradual and proper implementation of services that respect the constitutional legal regime of SUS, with no privileges or distortions. The 
objective of the article is to verify the acting of the Public Prosecutor`s Office on the oversight of the role that the state can and must exercise on SUS organization, in search for the whole population attendance, in accordance with constitutional and legal parameters. Thus, despite the municipalization of the health actions and services, it is essential that the state perform, in respect of the federal constitution and the organic law of health, the attributions of inductor of the regionalized organization and promoter of complementary health actions, in order to contibute for SUS consolidation, what should be object of control of the Public Prosecutor`s Office.

Keywords: Unified Health System; Public Prosecutor`s Office; right to healthcare.

SUMÁRIO: Introdução; 1. Responsabilidade do Estado no contexto da municipalização da saúde; 2. Redes de Atenção à Saúde (RAS): o papel do Estado; 3. O Contrato Organizativo da Ação Pública da Saúde (COAP); 4. O Estado nos três níveis de complexidade do sistema;Conclusão; Referências Bibliográficas.

\section{INTRODUÇÃO}

Criado pela Constituição Federal de 1988, que lhe dedicou um capítulo específico construído de acordo com o ideário da reforma sanitária brasileira defendido na Conferência Nacional de Saúde de 1986 (arts. 196 a 200, CF), o Sistema Único de Saúde (SUS) avançou consideravelmente em políticas destinadas a garantir o direito à saúde a todos que residem no Brasil, afastando o anterior modelo contributivo pelo qual somente trabalhadores com vínculo formal obtinham alguns tratamentos médico-hospitalares (art. 158, inciso XV, CF de 1967). Para tanto, ao longo dos anos o Poder Público, mediante articulação em seus três níveis federativos e contando com a participação complementar da iniciativa privada, vem implementando gradualmente uma rede pública de assistência em saúde gratuita, integral e universal, que atualmente alcança 150 milhões de pessoas que dependem dela exclusivamente (mais de $75 \%$ da população), a despeito das diferenças regionais e das características continentais do nosso país.

Ainda falta consciência sanitária aos brasileiros $^{1}$, que conhecem pouco a dimensão social do direito à saúde e o SUS, mas é possível apontar para vários avanços nas políticas públicas de saúde no Brasil, como a ampliação significativa do acesso da população às ações e serviços públicos de saúde, inclusive para aqueles que têm planos e seguros privados de saúde e procuram o SUS para as urgências e emergências, os medicamentos de alto custo e os procedimentos de alta complexidade ${ }^{2}$; as ações de saúde coletiva que atingem todos os brasileiros, como a vigilância sanitária, o combate de vetores, o controle de epidemias e endemias

\footnotetext{
${ }^{1}$ PAIM, Jairnilson Silva. O que é o SUS, Rio de Janeiro: Editora Fiocruz, 2009, p. 132.

${ }^{2} \mathrm{O}$ problema da interferência da saúde suplementar na saúde pública (SUS), inclusive por meio da perniciosa judicialização de demandas individuais descoladas das políticas públicas de saúde, não será abordado aqui por falta de espaço, mas a respeito do tema o volume 1 do Direito da Saúde no Brasil trouxe importantes reflexões, sobretudo os artigos de Lenir Santos e Marlon Alberto Weichert (Lenir Santos, org., Saberes Editora: Campinas, 2010). Sobre a judicialização da saúde de demandas privadas, em prejuízo da maioria da população, veja-se Judicialização da saúde: regime jurídico do SUS e intervenção na administração pública, de Reynaldo Mapelli Júnior, Rio de Janeiro: Atheneu, 2017; Ministério Público: atuação na área da saúde pública, de Reynaldo Mapelli Júnior, in "MINISTÉRIO PÚBLICO - Vinte e cinco anos do novo perfil constitucional", de Walter Paulo Sabella, Antônio Araldo Ferraz Dal Pozzo e José Emmanuel Burle Filho (coord.), São Paulo: Malheiros Editores, pp. 457-484, 2013; Claiming the Right to Health in Brazilian Courts: The Exclusion of the Already Excluded?, de Virgílio Afonso da Silva e Fernanda Vargas Terrazas, in Law \& Social Inquiry - Journal of The American Bar Foundation, 36 (4):825853, 2011; Harming the Poor Through Social Rights Litigation: Lessons from Brazil, de Octavio Luiz Motta Ferraz, in Texas Law Review, University of Texas: EUA, vol. 89:1643-1668, 2011; e Garantia do direito social à assistência farmacêutica no Estado de São Paulo, de Silvia Badim Marques e Sueli Galdolfi Dallari, in Rev. Saúde Pública, 41 (1):101-107, 2007.
} 
e os programas de imunização ${ }^{3}$; a política nacional de atenção básica e o seu Programa de Saúde da Família (PSF) com reconhecimento internacional, inclusive pela Organização Mundial de Saúde (OMS); políticas específicas como a de tratamento integral oncológico (quimioterapia, radioterapia, medicamentos, etc.), dos portadores de HIV/AIDS ${ }^{4}$, de Hepatite $\mathrm{C}$ e de outras doenças crônicas; e a realização de inúmeros procedimentos de alta complexidade, como os do sistema nacional de transplantes. ${ }^{5}$

Apesar disso, não há dúvida de que muitos problemas ainda persistem e que continua a luta pelo SUS, cujos princípios e diretrizes constitucionais de universalidade (art. 194, parágrafo I, único, e art. 196, caput, CF), integralidade (art. 198, II, CF), igualdade (art. 196, caput, CF), gratuidade (art. 222, V, da Constituição do Estado de São Paulo e art. 2º, I, da LC n 141/2012), regionalização e hierarquização (art. 198, caput, CF), descentralização (art. 198, I, CF), participação social (art. 194, parágrafo único, VII e art. 198, III, CF, e Lei no 8.142/ 90) e informação (artigo $7^{\circ}$, inciso VI, da Lei 8.080/90) dependem, como não poderia deixar de ser, da implementação gradual e adequada de serviços que respeitem o regime jurídico constitucional do SUS, sem privilégios e distorções. Ainda há falta de resolutividade na atenção básica, de equipamentos para atendimentos de maior complexidade em várias regiões do país, de recursos financeiros adequados, de planejamento para enfrentar o envelhecimento da população e o aumento de doenças crônicas, de modelos de gestão eficientes, recursos humanos adequados e controle social sem a nefasta dominação de interesses político-partidários como frequentemente vem ocorrendo. ${ }^{6}$

A organização da rede pública dos serviços sanitários, que deve expressar os princípios de regionalização, hierarquização e descentralização político-administrativa, na prática se tornou um dos principais desafios para a consolidação do SUS, pois, ainda que o usuário consiga o acesso às ações e serviços públicos de saúde ("portas de entrada"), isso não significa a resolução de suas demandas mais comuns (atenção básica ou primária) ou o automático referenciamento para unidades de saúde de maior complexidade, quando necessário para garantir a integralidade da assistência. Nesse assunto, como a saúde do indivíduo frequentemente depende de esfera de atuação que ultrapassa o espaço político-administrativo do Município, o Estado é que deve ser o condutor na organização regional de serviços, atentando para os três níveis de complexidade do atendimento. Infelizmente, porém, a ênfase na municipalização da saúde, induzida por uma normatização excessiva do Ministério da Saúde que pouco espaço deixou para a pactuação de

\footnotetext{
${ }^{3} \mathrm{Na}$ área de medicina preventiva, costuma-se citar como exemplo de sucesso a eliminação da poliomielite em 1989, a ausência de casos autóctones de sarampo desde 2000 e a vacina para gripe para idosos e pessoas com saúde debilitada.

${ }^{4}$ Com o fornecimento gratuito do coquetel de medicamentos anti-retrovirais para AIDS e campanhas de prevenção e diagnóstico precoce que levaram, dentre outras coisas, à diminuição da transmissão vertical da doença (mãe-filho) e da mortalidade, o programa de AIDS do Brasil é considerado o melhor do mundo pela Organização Mundial da Saúde (OMS).

${ }^{5}$ O sistema público de transplantes de órgãos do Brasil é o maior do mundo, com cerca de $40 \%$ dos transplantes realizados no Estado de São Paulo; na cidade de São Paulo, aliás, o Hospital Estadual do Rim e Hipertensão há 10 (dez) anos é o líder mundial de transplante renal.

${ }^{6}$ Todos esses problemas são preocupantes, mas dentre eles gostaríamos de destacar a diminuição dos recursos destinados aos SUS, que beira o escândalo: o Brasil é o $72^{\circ}$ país no ranking atual da Organização Mundial da Saúde (OMS) no gasto per capita em saúde, estando abaixo de alguns países africados e seus vizinhos Argentina, Uruguai e Chile; depois da perda dos recursos do antigo Instituto Nacional de Assistência e Previdência Social (INAMPS) em 1993, a esperança era a regulamentação da Emenda Constitucional $n^{\circ} 29$, de 13.9.2000, que veio com a Lei Complementar $\mathrm{n}^{\circ} 141$, de 13.1.2012, que não fixou percentual mínimo de recursos para a União, como existe para Estados e Municípios e estava previsto no projeto de lei ... As Emendas Constitucionais no 86/2015 95/2016, com o seu congelamento de recursos e teto para gastos, consolidaram de vez o desfinanciamento do SUS. Cf, para uma visão ampla do problema, O Financiamento da Saúde no Brasil, de Eurípides Balsanufo Carvalho (org.), São Paulo: Conselho Regional de Medicina do Estado de São Paulo (CREMESP), 2010, e Ministério Público: atuação na área da saúde pública, de Reynaldo Mapelli Júnior, ob. cit., pp. 473-474.
}

Revista de Direito Brasileira | São Paulo, SP | v. 18 | n. 7 | p. 315 - 330 |Set./Dez. 2017 
responsabilidades, empurrou os Estados para uma espécie de "elo perdido", na feliz expressão de Lenir Santos e Luiz Odorico Monteiro de Andrade:

O Estado, numa Federação, deve ser o condutor das políticas que exigem compartilhamentos de serviços, interdependência de serviços, como é o caso do SUS que exige sejam todos os serviços públicos de saúde integrados numa rede regionalizada e hierarquizada de saúde, constituindo um único sistema, nos termos do art. 198 da CF. Contudo, os Estados, com amplo poder-dever constitucional e legal de organização político-administrativa da sua região, vêm sendo o elo perdido nessa articulacão regional do SUS (g.n.). ${ }^{7}$

Por essa razão, o Ministério Público, instituição que o próprio texto constitucional escolheu como fiscalizador dos serviços de relevância pública (art. 129, inciso II, CF), ou seja, as ações e serviços de saúde, únicos qualificados como tal (art. 197, CF) ${ }^{8}$, a despeito do controle exercido sobre as atividades dos Municípios, deve se preocupar especialmente com o papel que o Estado pode e deve exercer na organização do SUS, em busca do atendimento integral da população, de acordo com os parâmetros constitucionais e legais, notadamente as alterações normativas ensejadas com a regulamentação da Lei Orgânica da Saúde (Decreto n ${ }^{\circ} 7.508$, de 28 de junho de 2011, que trata da organização do SUS, do planejamento da saúde, da assistência da saúde e da articulação interfederativa) e as últimas modificações na própria $\operatorname{LOS}^{9}$.

\section{RESPONSABILIDADE DO ESTADO NO CONTEXTO DA MUNICIPALIZAÇÃO DA SAÚDE}

Depois da Declaração de Alma-Ata, de 12 de setembro de $1978^{10}$, a atenção básica (ou primária) tornou-se o foco principal das políticas públicas de saúde, constituindo a porta de entrada do paciente no sistema público de saúde, local da assistência inicial e atendimento de suas principais necessidades, além de setor responsável pela organização do cuidado, propiciando o referenciamento para serviços de maior complexidade. No Brasil, os cuidados primários incluem a promoção de condições ambientais e nutricionais adequadas, a educação em saúde, o saneamento básico, os cuidados materno-infantis, o planejamento familiar, as imunizações, a prevenção e controle de doenças endêmicas, a disponibilização de exames preventivos e medicamentos essenciais, bem como, a cura e reabilitação das doenças mais comuns. ${ }^{11}$

Os cuidados primários e alguns serviços especializados vêm sendo progressivamente atribuídos aos Municípios brasileiros, por prevalecer o entendimento de que constituem o ente

\footnotetext{
${ }^{7}$ O Estado na articulação do Sistema de Saúde: um elo perdido?, artigo acessível na internet, inclusive no sítio www.idisa.org.br. [acesso em 17.10.2014].

${ }^{8}$ Defendendo que a caracterização das ações e serviços de saúde como de relevância pública teve como objetivo ensejar o controle do Ministério Público, veja-se O Conceito Constitucional de Relevância Pública, de Sueli Gandolfi Dallari (organizador), Brasília: Organização Pan-Americada da Saúde, 1992, notadamente p. 20; e Sistema Único de Saúde: comentários à Lei Orgânica da Saúde, de Guido Ivan de Carvalho e Lenir Santos, $4^{\mathrm{a}}$ edição revista e atualizada, Campinas: Editora da UNICAMP, 2006, p. 263.

${ }^{9}$ A Lei ${ }^{\circ}$ 12.401, de 28 de abril de 2011, que dispôs sobre a assistência terapêutica e a incorporação de tecnologias em saúde no SUS, e à Lei no 12.466, de 24 de agosto de 2011, que tratou dos foros de pactuação e negociação conhecidos como Comissão Intergestores Tripartite (CIT), Comissão Intergestores Bipartite (CIB), bem como, do Conselho Nacional de Secretários de Saúde (CONASS) e do Conselho Nacional de Secretarias Municipais de Saúde (CONASEMS).

${ }^{10}$ Conferência Internacional sobre Cuidados Primários de Saúde, antiga URSS, no período de 6 a 12.9 .1978$.

${ }^{11}$ Brasil. Política Nacional de Atenção Básica, Portaria MS no 2.488, de 21 de outubro de 2011. Para uma análise detalhada, inclusive do Programa de Saúde da Família (PSF) e das doenças negligenciadas, cf. Reynaldo Mapelli Júnior, Mário Coimbra e Yolanda Alves Pinto Serrano de Matos, Direito Sanitário, São Paulo: Ministério Público do Estado de São Paulo (MPSP) e Imprensa Oficial do Estado de São Paulo, 2012, pp. 50-60.
}

Revista de Direito Brasileira | São Paulo, SP | v. 18 | n. 7 | p. 315 - 330 | Set./Dez. 2017 
federativo mais próximo da população. De fato, eles estariam mais próximos da população para o acesso inicial e a organização do cuidado, teriam como identificar melhor as necessidades mais prementes, e garantir a participação da comunidade local nas políticas públicas de saúde, o chamado controle social.

A municipalização da saúde, porém, somente surgiu no Direito Sanitário com a Lei Orgânica de Saúde, e não veio descolada de outras diretrizes:

Art. $7^{\circ}$ As ações e serviços públicos de saúde e os serviços privados contratados ou conveniados que integram o Sistema Único de Saúde (SUS), são desenvolvidos de acordo com as diretrizes previstas no art. 198 da Constituição Federal, obedecendo ainda aos seguintes princípios:

IX - descentralização político-administrativa, com direção única em cada esfera de governo:

a) ênfase na descentralização dos serviços para os municípios; b) regionalização e hierarquização da rede de serviços de saúde.

A Constituição Federal não fez referência expressa à municipalização da saúde e criou um sistema de solidariedade passiva entre União, Estados, Distrito Federal e Municípios, todos com o dever de "cuidar da saúde" (art. 23, II, CF). É nesse contexto, que pressupõe o entendimento de que os gestores federal, estadual e municipal integram um sistema único, organizado em rede regionalizada e hierarquizada, é que deve ser entendida a "descentralização, com direção única em cada esfera de governo", um dos princípios organizacionais ou operativos do SUS (art. 197, caput e inciso I - os outros princípios são a integralidade e a participação da comunidade).

Como ensinam Sueli Gandolfi Dallari e Vidal Serrano Nunes Júnior:

A indicação constitucional (art. 198, I) não desonera quaisquer das instâncias federativas para com o dever de assistência à saúde (...). Apesar de se tratar de uma obrigação solidária, o que faz com que qualquer um dos entes possa ser demandado pela ausência de prestações em matéria de saúde, o sistema apresenta uma diretriz de descentralização, indicando a necessidade de que os serviços de atendimento seja objeto de um processo de municipalização. (...) Esse processo de descentralização também ocorre na relação entre União e os Estados, em uma dimensão evidentemente menos complexa pelo número relativamente pequeno de unidades federadas envolvidas (Estados e Distrito Federal). ${ }^{12}$

O problema da indefinição do papel dos Estados na organização do SUS está inserido no processo histórico de municipalização da saúde, geralmente promovida por intensa regulamentação do Ministério da Saúde que praticamente ignorou que a diretriz está relacionada com a regionalização e a hierarquização dos serviços, estando preservada a direção única em cada esfera de governo na descentralização política-administrativa.

${ }^{12}$ Direito Sanitário, São Paulo: Editora Verbatim, 2010, pp. 84-89.

Revista de Direito Brasileira | São Paulo, SP | v. 18 | n. 7 | p. 315 - 330 |Set./Dez. 2017 
Um gráfico com a linha do tempo com estas normas permite uma visão panorâmica desse processo:

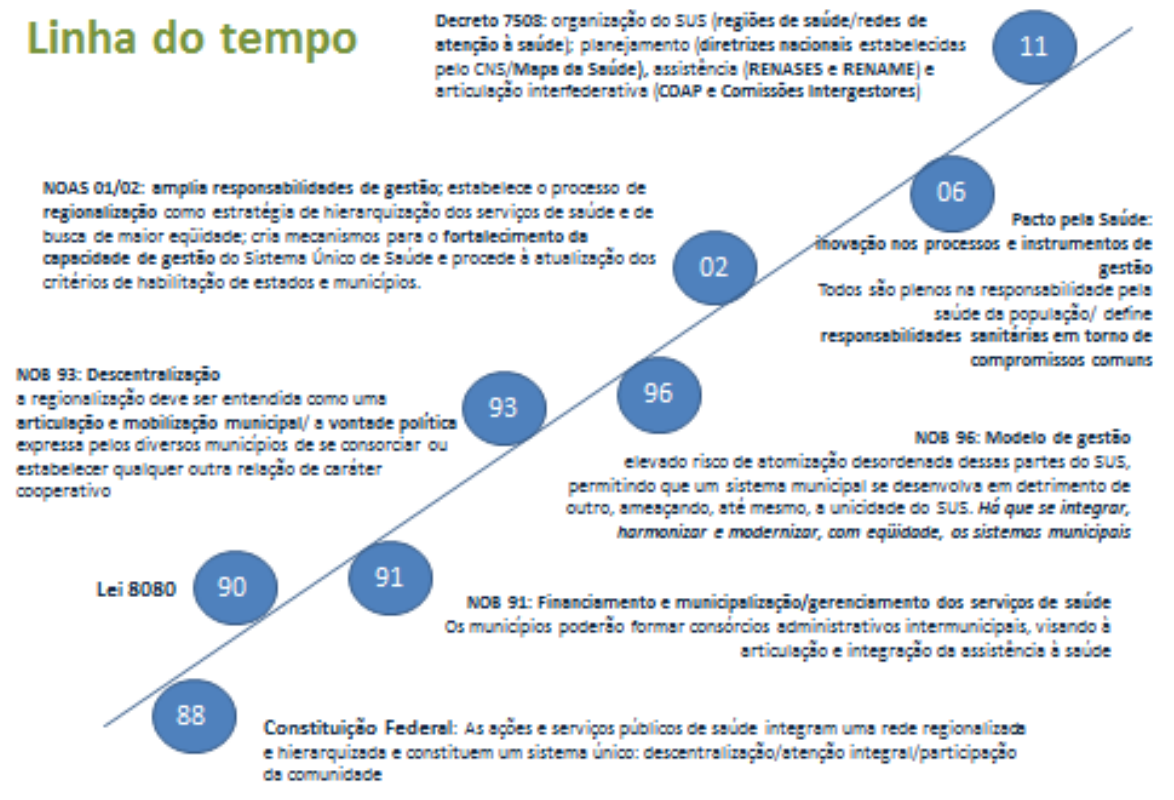

Fonte: Coordenadoria de Planejamento de Saúde (CPS) da SES/SP

A partir da Norma Operacional Básica (NOB) nº 01/93, que cuidou do gerenciamento da descentralização, tratou das Comissões Intergestores Tripartite e Bipartite (CIT e CIB) e criou sistemáticas de relacionamento entre os entes da federação para promover a autonomia progressiva e voluntária dos Municípios, o Ministério da Saúde passou a editar uma série de Normas Operacionais Básicas (NOB) e Normas Operacionais de Assistência à Saúde (NOAS), sempre focado no processo de descentralização político-administrativa do SUS. Quase sempre o fez, porém, na lógica da habilitação do Município para receber recursos federais e na ausência de um plano de regionalização e hierarquização de serviços.

A NOB no 01/96 chegou a reconhecer a possibilidade fragmentação do SUS pela falta de atuação dos Estados, ou seja, "o elevado risco de atomização desordenada dessas partes do SUS, permitindo que um sistema municipal se desenvolva em detrimento de outro, ameaçando, até mesmo, a unicidade do SUS". Apesar disso, pouco se fez para corrigir esta distorção, e a NOAS $n^{\circ}$ 01/2001, que foi revista pela NOAS no 01/2002 e instituiu o Plano Diretor de Regionalização para a organização de regiões e microrregiões de saúde, causou ainda mais confusão, parecendo confundir a direção única em cada esfera administrativa com o comando total de um Município sobre todos os serviços de saúde situados em sua região geográfica. ${ }^{13}$

Na prática, a responsabilidade do gestor municipal é fundamental, mas não é suficiente para garantir a integralidade da assistência, pois existem graus de capacidade administrativa dos Municípios, que não podem ser tratados igualitariamente, e serviços de outros Municípios e do Estado que devem ser disponibilizados para uma inteira região (frequentemente os de maior complexidade).

Houve uma tentativa de delimitação formal das responsabilidades sanitárias de cada um dos gestores do SUS no Pacto pela Saúde, assinado pelo Ministério da Saúde, Conselho Nacional

\footnotetext{
${ }^{13}$ Estas e outras críticas podem ser conferidas em O Papel do Gestor Estadual no Sistema Único de Saúde (SUS), de Luiz Roberto Barradas Barata, Oswaldo Yoshimi Tanaka e José Dínio Vaz Mendes, publicação da Secretaria de Estado da Saúde de São Paulo (SES/SP), 2003.
}

Revista de Direito Brasileira | São Paulo, SP | v. 18 | n. 7 | p. 315 - 330 | Set./Dez. 2017 
de Secretários de Saúde (CONASS) e Conselho Nacional de Secretários Municipais de Saúde (CONASEMS), mas dúvidas persistiram (Portaria Ministerial $\mathrm{n}^{\circ}$ 399, de 22 de fevereiro de 2006).

Seguiram-se, ainda, complementares Pactos pela Saúde em 2007, 2008, 2009 e 2010/2011 (Portarias Ministeriais $n^{\circ}$ 91, de 10 de janeiro de 2007, $n^{\circ} 325$, de 21 de fevereiro de 2008, n 48 , de 12 de janeiro de 2009 e $\mathrm{n}^{\circ} 2.669$, de 3 de novembro de 2009), mas o excesso da normatização infraconstitucional, com modificações constantes e confusas, ficou bastante claro quando foi publicado um Regulamento do Sistema Único de Saúde no Diário Oficial da União (Portaria Ministerial $n^{\circ} 2.048$, de 3 de setembro de 2009), uma pretendida "consolidação" das normas administrativas, com inacreditáveis 790 artigos e 94 anexos, espalhados por mais de 150 páginas. $^{14}$

O que se espera é que, com a publicação do Decreto $n^{\circ} 7.508$, de 28 de junho de 2011, que regulamenta a Lei Orgânica da Saúde em pontos fundamentais como o planejamento ascendente, a articulação interfederativa e a organização do SUS em regiões de saúde, esse panorama de incertezas e insegurança jurídica seja afastado gradualmente, mas para isso a atuação dos Estados será decisiva.

\section{REDES DE ATENÇÃO À SAÚDE (RAS): O PAPEL DO ESTADO}

A organização e o funcionamento do SUS, durante o período das normas operacionais básicas e até mesmo do pacto pela saúde, foram marcados pela imposição coercitiva dos programas federais, pela habilitação burocrática de Municípios e Estados em modelos de gestão para o recebimento de recursos federais obrigatórios por força de lei ${ }^{15}$ (gestão incipiente, plena, semiplena, plena da atenção básica, etc.) e pela normatização excessiva do Ministério da Saúde. Como resultado, houve fracionamento de recursos e políticas públicas, quase nunca vinculados a indicadores de saúde e satisfação dos usuários.

Sabe-se que a burocracia estatal e o excesso de normatização infralegal do Direito Administrativo, com suas intermináveis e cambiantes portarias, resoluções e atos administrativos, estão muito arraigados na cultura brasileira. O SUS é um exemplo significativo desse tipo de burocracia estatal, mas felizmente o surgimento de foros de negociação e pactuação entre gestores que devem compartilhar a administração pública em termos financeiros, logísticos e prestacionais, como as Comissões Intergestores Tripartite e Bipartite (CIT e CIB), o Conselho Nacional de Secretários da Saúde (CONASS) e o Conselho Nacional de Secretários Municipais de Saúde (CONASEMS), órgãos reconhecidos pela Lei n 12.466 , de 24 de agosto de 2011, levou a uma mudança de paradigma: pensa-se, agora, em respeitar a autonomia de cada ente federado (a "direção única em cada esfera de governo") e construir consensos interfederativos sobre as redes de serviços de saúde. À imposição de programas federais formulados unilateralmente, contrapõese a ideia de uma administração pública concertada.

$\mathrm{Na}$ realidade, o federalismo brasileiro é predominantemente cooperativo e consensual, por força das competências comuns e concorrentes estabelecidas pela Constituição Federal, especialmente perceptível no caso da conjugação de esforços para a prestação de serviços públicos essenciais. José dos Santos Carvalho Filho fala, nesse caso, em gestão associada dos

\footnotetext{
${ }^{14}$ Sobre isso, cf. Direito Sanitário, de Reynaldo Mapelli Júnior, Mário Coimbra e Yolanda Alves Pinto Serrano de Matos, op. cit., pp. 41-44.

${ }^{15}$ A respeito dos recursos financeiros, é preciso sempre relembrar que as transferências intergovernamentais foram previstas na Lei $\mathrm{n}^{\circ} 8.142 / 90$, segundo qual dever ser repassadas “de forma regular e automática para os Municípios, Estados e Distrito Federal" de acordo com os critérios da LOS (art. 3 ${ }^{\circ}$, caput), atualmente respeitando-se a Emenda Constitucional $n^{\circ}$ 29/200 e a Lei Complementar no 141/2012 que a regulamentou, não fazendo qualquer sentido os incontáveis pedidos que políticos e sociedade civil fazem aos gestores de saúde nos gabinete, como se tratasse de um liberalidade deles.
} 
entes federativos, defendida pelo moderno federalismo de cooperação, a ser formalizada em convênios de cooperação e consórcios públicos:

A Constituição, para deixar claro esse intento, previu, ao instituir a reforma administrativa do Estado (EC n $\left.{ }^{\circ} 19 / 1998\right)$, a gestão associada na prestação de serviços públicos, a ser implementada, através de lei, por convênios de cooperação e consórcios públicos celebrados entre a União, os Estados, Distrito Federal e os Municípios. Trata-se, como já tivemos a oportunidade de examinar, de instrumentos de cooperação visando a alcançar objetivos de interesses comuns dos pactuantes. A noção de gestão associada emana da própria expressão: significa uma conjugação de esforços visando a fins de interesse comum dos gestores. Em relação à gestão associada de serviços públicos, podese adotar a conceituação de que corresponde ao 'exercício das atividades de planejamento, regulação ou fiscalização de serviços públicos por meio de consórcio público ou de convênio de cooperação entre entes federados, acompanhadas ou não da prestação de serviços públicos ou da transferência total ou parcial de encargos, serviços, pessoal e bens essenciais à continuidade dos serviços transferidos'. ${ }^{16}$

Embora nem sempre o papel dos Estados-membros esteja claro na repartição constitucional de competências, Eugênio Vilaça Mendes adverte acerca da importância do ente estatal:

No modelo federativo tripartite brasileiro, a gestão das políticas públicas tornase mais complexa na medida em que é preciso conjugar a interdependência e autonomia dos três entes. A cooperação federativa, por sua vez, precisa ser exercida tanto pela União quanto pela esfera estadual em relação aos municípios. Ainda que a esfera estadual seja aquela com atribuições menos definidas na Constituicão Federal, cabe a ela um papel central nas políticas sociais, particularmente quando se considera a heterogeneidade dos municípios em termos de capacidade técnica, administrativa e financeira (g.n.). ${ }^{17}$

No caso da saúde pública, a cooperação dos gestores do SUS decorre da própria Constituição Federal, como ensinam Lenir Santos e Luiz Odorico Monteiro de Andrade:

O artigo 198 da CF ao dispor que 'as ações e serviços de saúde integram uma rede regionalizada e hierarquizada e constituem um sistema único', conforma uma rede interfederativa de serviços que exige interação, integração e articulação de todos os entes federados. (...) Por tudo isso, e sendo o SUS uma rede interfederativa de serviços em decorrência da integralidade da assistência que o conforma como 'rede', somente decisões consensuais entre os entes federativos implicados nesse fazer estão legitimadas para definir, em detalhes, as responsabilidades destes na saúde. ${ }^{18}$

\footnotetext{
${ }^{16}$ Manual de direito administrativo, $25^{\mathrm{a}}$ ed. rev., ampl. e atual. até a Lei $\mathrm{n}^{\circ} 12.587$, de 3.1.2012, São Paulo: Atlas, 2012, pp. 351-352. A conceituação foi retirada do Decreto $\mathrm{n}^{\circ}$ 6.017, de 17.1.2007, que regulamentou a Lei $\mathrm{n}^{\circ}$ 11.107/2005, disciplinadora dos consórcios públicos.

${ }^{17}$ As redes de atenção à saúde, Brasília/DF: Organização Pan-Americana da Saúde, 2011, p. 162.

${ }^{18}$ SUS: o espaço da gestão inovadora e dos consensos interfederativos: aspectos jurídicos, administrativos $e$ financeiros, $2^{a}$ ed., Campinas/SP: Saberes Editora, 2009, pp. 109-110. A ideia de gestão compartilhada consensual é defendida pelos autores citando Odete Medauar, $O$ direito administrativo em evolução, $2^{\text {a }}$ edição, São Paulo: ed. RT, 2003.
} 
Como os Municípios gerenciam apenas os serviços que estão sob sua gestão e formulam as políticas públicas destinadas à população de seu território, na prática a organização da rede de serviços do SUS ocorre regionalmente, em negociação nos colegiados regionais que reúnem Secretaria de Estado da Saúde e Secretarias Municipais de Saúde, em um planejamento ascendente que parte do município, segue pela região e se conforma com as diretrizes nacionais. Sob a liderança dos Estados, que formulam a política estadual/regional e articulam com os Municípios, são definidas as portas de entrada, os serviços públicos e privados disponíveis, os de maior complexidade sob gestão estadual e federal, a regulação regional das vagas e o sistema de cofinanciamento (União, Estados e Municípios).

Segundo o Decreto $n^{\circ} 7.508 / 11$, “o acesso universal, igualitário e ordenado às ações e serviços de saúde se inicia pelas Portas de Entrada do SUS e se completa na rede regionalizada e hierarquizada, de acordo com a complexidade do serviço" (art. $8^{\circ}$ ). As redes interfederativas de serviços, responsáveis, assim sendo, pelo tratamento integral (em níveis de complexidade crescente) e completo (do ingresso obrigatório do usuário no SUS até os atendimentos mais complexos) do paciente, foram denominadas Redes de Atenção à Saúde (RAS), têm a "finalidade de garantir a integralidade da assistência à saúde" (at. $2^{\circ}$. VI) e devem ser "instituídas pelo Estado, em articulação com os Municípios, respeitadas as diretrizes gerais pactuadas na Comissão Integestores Tripartite - CIT" (art. $4^{\circ}$.).

A configuração do desenho estrutural do SUS, como uma malha organizada e hierarquizada de serviços, portanto, é tarefa precípua do Estado. No fundo, volta-se para a concepção de sistema da Constituição Federal (art. 198), tantas vezes ignorada pela judicialização da saúde, que se traduz na organização de um conjunto de ações e serviços públicos de saúde, com acesso regulado e um sistema de referência e contrarreferência. O Decreto diz quais são as portas de entrada do sistema, as ações e serviços que devem obrigatoriamente ser ofertadas pela rede regional de saúde e o referenciamento para ambulatórios de especialidades e hospitais:

Art. 5. Para ser instituída, a Região de Saúde deve conter, no mínimo, ações e serviços de:

I - atenção primária;

II - urgência e emergência;

III - atenção psicossocial;

IV - atenção ambulatorial especializada e hospitalar; e

V - vigilância em saúde.

Art. $9^{\circ}$. São Portas de Entrada às ações e aos serviços de saúde nas Redes de Atenção à Saúde os serviços:

I - de atenção primária;

II - de atenção de urgência e emergência;

III - de atenção psicossocial;

IV - especiais de acesso aberto.

Art. 10. Os serviços de atenção hospitalar e os ambulatoriais especializados, entre outros de maior complexidade e densidade tecnológica, serão referenciados pelas Portas de Entrada de que trata o art. $9^{\circ}$.

$\mathrm{Na}$ articulação regional de Municípios de uma determinada localidade, com vistas a instituir uma Rede de Atenção à Saúde (RRAS), também conhecida como Rede Regional de Atenção à Saúde (RRAS), em consonância com as diretrizes nacionais da União (art. 14), o Estado deve observar:

a) o respeito à autonomia dos Municípios, mas com eles articulando as formas de solução das demandas regionais; 
b) a atribuição da atenção básica a todos os Municípios (unidades básicas de saúde, programa de saúde de família, pronto-socorro, vigilância sanitária municipal, etc.), cabendo àqueles de maior porte parte da assistência de maior complexidade;

c) o respeito à atenção básica como porta de entrada (juntamente com a urgência/emergência e os Centros de Atenção Psicossocial/CAPS para a assistência em saúde mental), ordenadora da atenção e orientadora da rede;

d) o estímulo à lógica da necessidade e não da oferta do prestador, item para o qual o mapa da saúde se mostra instrumento muito útil;

e) o fortalecimento dos Departamentos Regionais de Saúde das Secretarias de Estado (DRS) e dos Colegiados de Gestão Regional (CGR);

f) a organização da regulação de vagas e cogestão da assistência, por vezes tomando para si a regulação regional, por vezes repassando-a para os Municípios desde que estejam de acordo e sejam treinados e submetidos à fiscalização do Estado;

g) o auxílio estatal na qualificação dos serviços municipais, no cofinanciamento em diversas hipóteses e na avaliação contínua de sua eficiência e da satisfação do usuário;

h) a prestação direta de alguns serviços, em geral aqueles de média e alta complexidade que estão a cargo de ambulatórios médicos de especialidades (AME) e dos hospitais universitários, como forma de se garantir regionalmente a integralidade da assistência.

Um exemplo exitoso da regionalização prevista no Decreto $\mathrm{n}^{\circ}$ 7.508/11 ocorreu no Estado de São Paulo, pois desde 2011 a Secretaria de Estado da Saúde conduziu um processo democrático de discussão com os Municípios paulistas, construindo consensualmente os mapas de saúde (art. 17), em um planejamento ascendente que foi ao final pactuado nos 63 Colegiados Regionais de Saúde (CRS) e na Comissão Intergestores Bipartite (CIB), sempre tendo como pano de fundo o plano de saúde de cada ente federado e a programação anual. ${ }^{19}$ Um gráfico nos permite verificar como ficaram as 17 Redes Regionais de Atenção à Saúde (RRAS), no Estado mais complexo da Federação:

\footnotetext{
${ }^{19}$ Nesse trabalho, foram considerados sobretudo os seguintes instrumentos de gestão: a) o plano de saúde, em consonância com o plano plurianual/PPA (art. 35, §2 $2^{\circ}$ I, ADCT, CF); b) a programação anual de saúde (art. 36, §2º LC n ${ }^{\circ} 141 / 2012$, e art. 35, §2º II, ADCT, CF ); c) o relatório de gestão (art. 36, $\S 1^{\circ}$, LC n $^{\circ} 141 / 2012$ e art. $3^{\circ}$ Portaria $\mathrm{GM} / \mathrm{MS} \mathrm{n} \mathrm{n}^{\mathrm{0}}$ 575/12). A orientação sempre foi a de atentar para a avaliação e o controle desse planejamento a cargo dos Conselhos de Saúde, dos Tribunais de Contas e do Ministério Público, na forma estabelecida pelo art. 38 da LC $\mathrm{n}^{\circ} 141 / 12$.
} 


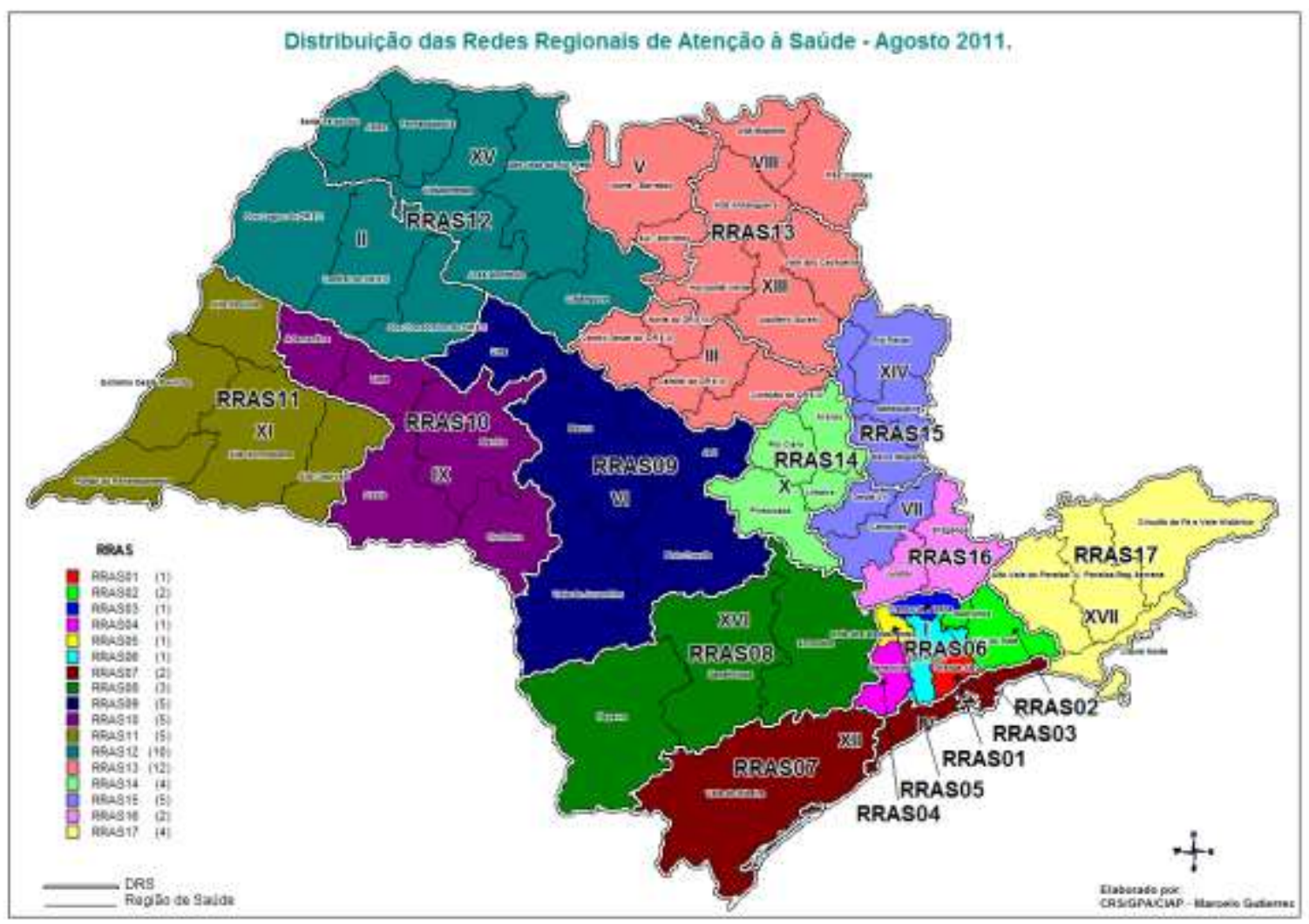

Fonte: Coordenadoria de Regiões de Saúde (CRS) da SES/SP

\section{O CONTRATO ORGANIZATIVO DA AÇÃO PÚBLICA DA SAÚDE (COAP)}

O planejamento regional do SUS, com a elaboração do mapa de saúde com os serviços públicos e privados de determinada região e o vazio assistencial identificável, e a integração orgânica dos diversos instrumentos de gestão (especialmente os planos municipal, estadual e nacional), possibilitou, assim sendo, a formação das redes regionais de saúde que devem garantir, por determinação constitucional (art. 198, II, CF), a integralidade da assistência, "em todos os níveis de complexidade do sistema" (art. $7^{\circ}$, II, LOS).

Esse sistema somente terá sentido se for coroado pelo Contrato Organizativo da Ação Pública da Saúde (COAP), instrumento jurídico por meio do qual os entes federados formalizam o acordo de vontades sobre a implementação, a execução e o financiamento das ações e serviços de saúde em uma região (arts. 33 e 34 do decreto). Trata-se, a rigor, de um novo instrumento jurídico a integrar o Direito Administrativo, que já está sendo implementado progressivamente em regiões do país ${ }^{20}$, para o qual logo de início Reynaldo Mapelli Júnior, Mário Coimbra e Yolanda Alves Pinto Serrando de Matos teceram palavras elogiosas:

Outro avanço no ordenamento jurídico sanitário é a previsão do Contrato Organizativo da Ação Pública da Saúde, apesar da inexatidão do termo (não se trata de relação contratual, mas de gestão compartilhada de corresponsáveis), ato formal que deve registrar o acordo de colaboração dos gestores (art. 33), dentro de parâmetros legais (arts. 36 e 37), algo que, ao lado das Regiões de Saúde, propiciará maior organização do sistema e publicidade de suas virtudes e

\footnotetext{
${ }^{20}$ De acordo com o Ministério da Saúde, até agora foram entabulados COAPs nos Estados do Ceará, Alagoas e
} Matro Grosso do Sul. Cf. em www.portarsaude.saude.gov.br [acesso em 03.03.2014]. 
deficiências. Talvez, nesse ponto, vejamos o surgimento de uma nova 'judicialização', mais voltada para o cumprimento das pactuações. ${ }^{21}$

Defendidos por especialistas como Lenir Santos, para quem "o elo, o elemento aglutinador das responsabilidades, o estatuto jurídico que conformará as redes de atenção à saúde da região, com fixação das responsabilidades federativas na saúde, será o contrato", (...) "o qual terá a nobre função de dar garantia à conformação da rede de atenção à saúde e às responsabilidades dos entes nessa rede, será o elemento que possibilitará que a descentralização se regionalize"22, os COAPs somente se concretizarão se houver interesse e dedicação dos Estados, que precisam fornecer subsídios técnicos e de convencimento para a adesão dos Municípios, segundo as diretrizes nacionais da União ${ }^{23}$.

Espera-se, assim, que após as fases de elaboração dos mapas de saúde e instituição das redes regionais de atenção à saúde, os Estados exerçam o seu protagonismo na formalização dos consensos regionais em COAPs, no qual constará a responsabilidade de cada gestor na execução e no cofinanciamento das ações e serviços de sáude, o sistema de controle e auditoria e a cominação de sanções administrativas para o caso de inadimplência, um avanço significativo em termos de organização e transparência do SUS.

\section{O ESTADO NOS TRÊS NÍVEIS DE COMPLEXIDADE DO SISTEMA}

Afigura-se interessante registar alguns aspectos da atuação prática do gestor estadual no SUS na organização da rede regional de serviços, fazendo-o separadamente em relação à atenção básica e à atenção secundária e terciária. ${ }^{24}$

\section{Atenção Primária (básica)}

A atenção primária ou básica constitui o primeiro contato com o sistema de saúde, basicamente na unidade básica de saúde (UBS) ou por meio do Programa de Saúde da Família (PSF), bem como, nos Centros de Atenção Psicossocial (CAPS), por meio da qual deve ser resolvida a maioria dos agravos de saúde e organizado o cuidado do usuário ${ }^{25}$. Embora a atenção primária esteja sob a gestão municipal, o Estado deve se dedicar a ela porque a sua falta de resolutividade sobrecarrega os equipamentos de maior complexidade, existem grandes diferenças entre os Municípios que precisam ser enfrentadas com medidas de equidade e a organização regional pressupõe o controle das portas de entrada.

Assim, é preciso implementar um sistema de acompanhamento e avaliação da atenção primária, buscando conhecer e corrigir as falhas nas suas atividades e auxiliar os Municípios mais humildes, para que todos os usuários do Estado sejam adequadamente atendidos.

\footnotetext{
${ }^{21}$ Direito Sanitário, op. cit., p. 36.

${ }^{22}$ Sistema Único de Saúde: os desafios da gestão interfederativa, Campinas/SP: Saberes Editora, 2013, p. 171, No mesmo sentido, Lenir Santos e Luiz Odorico Monteiro de Andrade, em SUS: o espaço da gestão inovadora e dos consensos interfederativos: aspectos jurídicos, administrativos e financeiros, op. cit., p. 151.

${ }^{23}$ Sobre elas, o Ministério da Saúde publicou o Guia para a elaboração do CONTRATO ORGANIZATIVO DA AÇÃO PÚBLICA: Construindo o COAP passo a passo, Série Articulação Interfederativl. 2, Odorico Monteiro de Andrade (coord.), Brasília/DF, 2013.

${ }^{24}$ Não ignoro a crítica de alguns especialistas que, ao invés dos três níveis de assistência, preferem "redes poliárquicas de atenção à saúde, em que, respeitando-se as diferenças tecnológicas, rompem-se as relações verticalizadas, conformando-se redes policêntricas horizontais", como afirma Eugênio Vilaça Mendes, no seu As redes de atenção à saúde,op. cit., p. 84 (com figuras ilustrativas dos dois sistemas). O preciosismo técnico, porém, não parece ter reflexos na prática.

${ }^{25}$ BRASIL. CONSELHO NACIONAL DE SECRETÁRIOS DE SAÚDE. Atenção Primária e Promoção da Saúde, Coleção Para Entender a Gestão do SUS, vol. 3, Brasília/DF: CONASS, 2011, pp. 10-37.
} 
Isso exige também, como base em indicadores de saúde e social (o Índice de Desenvolvimento Humano/IDH pode ser muito útil), o repasse inteligente de recursos estaduais para a assistência e para a reforma de equipamentos como as Unidades Básicas de Saúde, e

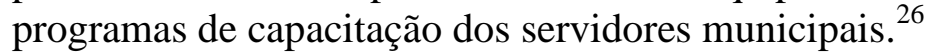

As vigilâncias sanitária e epidemiológica do Estado, além disso, devem fornecer capacitação técnica e apoio permanente aos colegas municipais, diante do ínfimo sucesso até agora obtido com a municipalização da vigilância, por falta de conhecimento técnico ou pela pressão política local, muitas vezes acompanhando in loco a vistoria dos fiscais municipais. $\mathrm{O}$ mesmo raciocínio serve para a regulação regional das vagas, a cargo do Estado ou de Municípios, neste último caso treinados e fiscalizados pelo gestor estadual. ${ }^{27}$

Por fim, cumpre ao gestor estadual formular e induzir políticas públicas que possam aprimorar a atenção primária e ter impacto em toda a rede de serviços, para maior resolutividade no primeiro nível de atendimento, como se tem feito, por exemplo, em diversas partes do país nas políticas de saúde mental, que não podem se resumir na criação de Centros de Atenção Psicossocial (CAPS), com a implementação de assistência especializada em saúde mental em prontos-socorros, ambulatórios e outros equipamentos inovadores.

\section{Atenção Secundária e Terciária}

A assistência de média e alta complexidade do SUS está mais próxima do Estado, que nesse campo assume frequentemente a função de prestador em ambulatórios de especialidades, hospitais gerais ou hospitais especializados ou de referência, embora isso varie muito em razão das demandas regionais e da capacidade de assistência dos Municípios. Nesse caso, o Estado precisa impulsionar o processo de regionalização e hierarquização sem ter medo de reestruturar todo o sistema em uma determinada região, modificando o papel e as funções de serviços, estes muitas vezes com taxas de subocupação e a população mal atendida, de acordo com critérios epidemiológicos regionais e indicadores técnicos. Fugindo, assim, da antiga lógica de oferta de serviços ou do pedido político, conseguirá contribuir para a integralidade da assistência, inclusive com os serviços sob sua gestão.

A principal tarefa do Estado passa a ser articulação entre os Municípios de uma determinada RAS e a oferta de serviços estaduais, para a instituição de um fluxo de referência e contrarreferência que disponibilize aos usuários os serviços especializados, de apoio diagnóstico e terapêutico, ambulatorial e hospital, as urgências e emergências, até mesmo os ambulatórios e hospitais altamente especializados (de nível terciário, normalmente estão vinculados a universidades estaduais e federais e atendem uma macrorregião). ${ }^{28}$

O papel político-institucional do Estado, nesse campo, deve ser o de coordenador do processo de planejamento regional, fortalecendo a participação ativa de todos os Municípios nos espaços regionais de discussão e planejamento, tomando cuidado para que Municípios menores ou sem representação político-partidária tenham voz, bem como, propondo formas de incentivo técnico e financeiro.

Os mecanismos para a regionalização e hierarquização incluem o acompanhamento constante dos serviços existentes e o incentivo para corrigir os vazios assistenciais (com

\footnotetext{
${ }^{26}$ No Estado de São Paulo, por exemplo, existe financiamento estadual para a atenção básica dos municípios (considerando o Piso de Atenção Básica/PAB e os municípios mais carentes), para os medicamentos essenciais (Programa Dose Certa), um programa de repasse de recursos para a reforma de Unidades Básicas de Saúde (UBS) e capacitação técnica em centros de referência normalmente vinculados a universidades.

${ }^{27}$ A Central de Regulação de Ofertas de Serviços de Saúde (CROSS) da Secretaria de Estado da Saúde de São Paulo regula os serviços estadual e regional, em um moderno sistema informatizado, e tem auxiliado diversos gestores municipais.

${ }^{28}$ BRASIL. CONSELHO NACIONAL DE SECRETÁRIOS DE SAÚDE. Assistência de Média e Alta Complexidade no SUS, Coleção Para Entender a Gestão do SUS, vol. 4, Brasília/DF: CONASS, 2011, pp. 10-47.
}

Revista de Direito Brasileira | São Paulo, SP | v. 18 | n. 7 | p. 315 - 330 |Set./Dez. 2017 
equipamentos municipais, estaduais ou mistos), inclusive com a imposição de metas e a avaliação sobre a eficiência do serviço prestado, o investimento em hospitais já existentes com perfil para a atuação regional $^{29}$, a contratação de serviços complementares privados se necessário, a criação de centros de referência para atendimentos especializados ${ }^{30}$, e a inserção efetiva das organizações sociais (OS), das organizações da sociedade civil de interesse público (OSCIP) e dos hospitais universitários no SUS, promovendo a regulação regional de forma adequada.

Em suma, o Estado não pode se omitir em ajudar os Municípios, sobretudo aqueles mais humildes, na oferta de serviços regionais e de referência, frequentemente mais complexos e caros, como a terapia renal substitutiva, a quimioterapia e a radioterapia, os exames especializados (tomografia, ressonância magnética, etc.), as unidades de terapia intensiva, o atendimento a gestantes e neonatos de alto risco, as cirurgias de maior complexidade e em muitos casos a própria emergência e urgência.

\section{CONCLUSÃO}

Em conclusão, pode-se afirmar que, ao contrário do que alguns pensam, o Estado tem um papel fundamental no SUS como planejador, organizador e prestador de serviços, com especial relevância na condução do planejamento ascendente e regional que deve levar à instituição das redes interfederativas de serviços, objetivando, ao final, a formalização do Contrato Organizativo da Ação Pública da Saúde (COAP) previsto no Decreto n 7.508/11.

Como fiscalizador do SUS, cabe ao Ministério Público, para além da costumeira atuação no controle de eventuais desvios da rede municipal de serviços de saúde, promover medidas que exijam do Estado o cumprimento de suas atribuições sanitárias, ensejando pactuação para a organização regionalizada e apoio complementar sempre que necessário, em respeito ao regime jurídico constitucional do SUS.

\section{REFERÊNCIAS BIBLIOGRÁFICAS}

BARATA, Luiz Roberto Barradas, TANAKA, Oswaldo Yoshimi e MENDES, José Dínio Vaz. $O$ Papel do Gestor Estadual no Sistema Único de Saúde (SUS), publicação da Secretaria de Estado da Saúde de São Paulo (SES/SP), 2003.

BRASIL. MINISTÉRIO DA SAÚDE / SECRETARIA DE GESTÃO ESTRATÉGICA E PARTICIPATIVA (SGEP) e DEPARTAMENTO DE ARTICULAÇÃO INTERFEDERATIVA (DAI). Guia para a elaboração do CONTRATO ORGANIZATIVO DA AÇÃO PÚBLICA: Construindo o COAP passo a passo, Série Articulação Interfederativa, vol. 2, Odorico Monteiro de Andrade (coordenador), Brasília/DF: Ministério da Saúde, 2013.

\footnotetext{
${ }^{29}$ Vale registrar que, diante da importância das Santas Casas de Misericórdia e dos ínfimos recursos federais que lhes são destinados, a Secretaria de Estado da Saúde de São Paulo, além de ter um programa de repasse de recursos estaduais complementares, desenvolve um programa de identificação dos hospitais filantrópicos estruturantes para uma determinada região, para repasses de aportes financeiros mais significativos objetivando redesenhar suas funções e atender melhor a região.

${ }^{30}$ Em São Paulo, a Secretaria de Estado da Saúde mantém 51 Ambulatórios Médicos de Especialidades, para consultas e exames especializados e procedimentos cirúrgicos de média complexidade, e tem centros de referência para tratamentos mais especializados e treinamento dos profissionais de saúde do SUS, como a Rede de Habilitação Lucy Montoro para pessoas com deficiência física (em parceria com a Secretaria de Estado dos Direitos da Pessoa com Deficiência), a Rede Hebe Camargo de Combate para tratamento oncológico e os Centros de Referência do Idoso (CRI).
} 
BRASIL. CONSELHO NACIONAL DE SECRETÁRIOS DE SAÚDE. Coleção Para Entender a Gestão do SUS, 13 volumes, Brasília/DF: CONASS, 2011. Pode ser acessado em www.conass.org.br.

CARVALHO, Eurípides Balsanufo (organização). O Financiamento da Saúde no Brasil: um debate sobre a vinculação constitucional de recursos para a saúde, dívida pública e projeto de reforma tributária, São Paulo: Conselho Regional de Medicina de São Paulo (CREMESP), 2010.

CARVALHO, Guido Ivan de e SANTOS, Lenir. Sistema Único de Saúde: comentários à Lei Orgânica da Saúde, 4ª edição revista e atualizada, Campinas: Editora da UNICAMP, 2006.

CARVAlHO FILHO, José dos Santos. Manual de direito administrativo, $25^{\mathrm{a}}$ ed. rev., ampl. e atual. até a Lei no 12.587, de 3.1.2012, São Paulo: Atlas, 2012.

DALlARI, Sueli Gandolfi (organizador). O Conceito Constitucional de Relevância Pública, Brasília: Organização Pan-Americana da Saúde, 1992.

DALLARI, Sueli Gandolfi e NUNES JÚNIOR, Vidal Serrano. Direito Sanitário, São Paulo: Editora Verbatim, 2010.

FERRAZ, Octavio Luiz Motta. Harming the Poor Through Social Rights Litigation: Lessons from Brazil, in Texas Law Review, University of Texas: EUA, vol. 89:1643-1668, 2011.

MAPELLI JÚNIOR, Reynaldo, COIMBRA, Mário e MATOS, Yolanda Alves Pinto Serrano de. Direito Sanitário, São Paulo: Ministério Público do Estado de São Paulo (MPSP) e Imprensa Oficial do Estado de São Paulo, 2012.

. Ministério Público: atuação na área da saúde pública, in "MINISTÉRIO PÚBLICO - Vinte e cinco anos do novo perfil constitucional”, Walter Paulo Sabella, Antônio Araldo Ferraz Dal Pozzo e José Emmanuel Burle Filho (coordenadores), São Paulo: Malheiros Editores, 2013, pp. 457-484.

Judicialização da saúde: regime jurídico do SUS e intervenção na administração pública, Rio de Janeiro: Atheneu, 2017.

MARQUES, Silvia Badim e DALLARI, Sueli Gandolfi. Garantia do direito social à assistência farmacêutica no Estado de São Paulo, in Rev. Saúde Pública, 41 (1):101-107, 2007.

MEDAUAR, Odete. O direito administrativo em evolução, $2^{\mathrm{a}}$ ed., São Paulo: Ed. RT, 2003.

MENDES, Eugênio Villaça. As redes de atenção à saúde, Brasília: Organização Pan-Americana de Saúde, 2011.

SANTOS, Lenir. Sistema Único de Saúde: os desafios da gestão interfederativa, Campinas/SP: Saberes Editora, 2013. 
SANTOS, Lenir e ANDRADE, Luiz Odorico Monteiro de. SUS: o espaço da gestão inovadora e dos consensos interfederativos: aspectos jurídicos, administrativos e financeiros, $2^{\mathrm{a}}$ ed., Campinas/SP: Saberes Editora, 2009.

- O Estado na articulação do Sistema de Saúde: um elo perdido?, artigo acessível na internet, inclusive no sítio www.idisa.org.br. [acesso em 1.3.2014]. SILVA, Silvio Fernandes da Silva (organizador). Redes de atenção à saúde no SUS: o pacto pela saúde e redes regionalizadas de ações e serviços de saúde, Gilson Carvalho... [et. al.], $2^{\mathrm{a}}$ ed, Campinas/SP: Saberes Editora, 2011.

SILVA, Virgílio Afonso da e TERRAZAS, Fernanda Vargas. Claiming the Right to Health in Brazilian Courts: The Exclusion of the Already Excluded?, in Law \& Social Inquiry - Journal of The American Bar Foundation, 36 (4):825-853, 2011.

WEICHERT, Marlon Alberto. Saúde e Federação na Constituição Brasileira, Rio de Janeiro: Lumen Juris, 2004. 